\title{
Synthesis and characterization of Ag nanoparticles and
}

\section{Ag loaded $\mathrm{TiO}_{2}$ photocatalysts}

\author{
G. Sauthier, ${ }^{1}$ A. Pérez del Pino, ${ }^{2}$ A. Figueras, ${ }^{1}$ E. György ${ }^{1,3^{*}}$
}

${ }^{1}$ Consejo Superior de Investigaciones Cientificas, Centre d'Investigacions en Nanociència i Nanotecnologia, (CIN2-CSIC), Campus UAB, 08193 Bellaterra, Spain

${ }^{2}$ Consejo Superior de Investigaciónes Científicas, Instituto de Ciencia de Materiales, (ICMAB-CSIC) Campus de la UAB, 08193 Bellaterra, Spain

${ }^{3}$ National Institute for Lasers, Plasma and Radiations Physics, P. O. Box MG 39, 77125 Bucharest, Romania

\begin{abstract}
$\mathrm{Ag}$ nanoparticles and $\mathrm{Ag}-\mathrm{TiO}_{2}$ nanostructures were grown on (001) $\mathrm{SiO}_{2}$ quartz substrates by pulsed laser deposition using a $\mathrm{KrF}^{*}$ excimer laser source $(\lambda=248 \mathrm{~nm}$, $\tau_{\mathrm{FWHM}} \sim 10 \mathrm{~ns}, v=10 \mathrm{~Hz}$ ) for the irradiation of $\mathrm{Ag}$ and $\mathrm{TiO}_{2}$ targets. The obtained structures were characterized by atomic force microscopy, X-ray diffraction, X-ray photoelectron spectroscopy, and UV-visible absorption spectroscopy. Photocatalytic activity was evaluated under near-UV light illumination by decomposition of methylene blue. The relationship between the laser synthesis process parameters and nanoparticles' chemical composition, crystal structure, morphology, shape and size distribution on the substrate surface were investigated and correlated with their optical properties. The established experimental parameters were used for the synthesis of nanostructures consisting of anatase phase $\mathrm{TiO}_{2}$ thin films covered by Ag nanoparticles for photocatalytic applications. The optimum experimental conditions were established which ensure significantly improved photoactivity in the presence of $\mathrm{Ag}$ nanoparticles as compared to the bare oxide surface.
\end{abstract}

\footnotetext{
"Corresponding author: Tel. +34935813725; Fax +34935813717; E-mail eniko.gyorgy@cin2.es
} 


\section{Introduction}

Noble metal nanoparticles represent subject of extensive research due to their potential applications in many key technological areas. ${ }^{1-3}$ In particular, silver nanoparticles owing to their characteristic strong surface plasmon resonance absorption in the visible range of the electromagnetic spectrum attracted huge interest in the last few years, for development of new type of biomedical, ${ }^{4-7}$ or nanoscale electronics and photonics ${ }^{8-12}$ devices. Many of these applications require the nanoparticles to be embedded or supported on a solid substrate. Moreover, the extreme sensitivity of the optical properties to the nanoparticles' geometry as size, shape, interparticle distance stands at the basis of all applications mentioned above. Therefore, the investigation of the relationship between the synthesis process parameters and nanoparticles' geometry is necessary to achieve controlled growth.

A large number of methods for the preparation of noble metal nanoparticle colloidal solution have been developed, including chemical processing ${ }^{13,14}$ or laser ablation of noble metal plates immersed in organic solvents. ${ }^{15-18}$ Homogeneous noble metal nanoparticle arrays were created on solid substrates by nanosphere lithography, ${ }^{6,19}$ electron beam lithography, ${ }^{20}$ and laser techniques. ${ }^{21,22}$ Oxide-noble metal nanocomposite thin films were synthesised of by sol-gel method, ${ }^{23} \mathrm{RF}$ sputtering, ${ }^{24,25}$ atom beam sputtering, ${ }^{26}$ or pulsed laser deposition. ${ }^{27-29}$

The sequential character of techniques including pulsed laser radiation permits the precise control of the amount of material deposited on the substrate surface. The possibility to grow multistructures consisting of subsequent deposition of different materials by the simple change of the targets submitted to the laser radiation stands also among the advantages of these methods.

Noble metal nanoparticles exhibit strong UV-visible absorption due to their plasmon resonance, which is produced by the collective oscillations of surface electrons. ${ }^{1-3}$ Their photosensitivity is potentially applicable to the development of new class of photocatalysts or photovoltaic fuel cells to meet the requirements of future environmental and energy technologies, driven by solar energy. 
The first objective of our investigations is the study of the evolution of the shape and size of $\mathrm{Ag}$ particles grown by pulsed laser deposition on (001) $\mathrm{SiO}_{2}$ quartz substrates, as a function of increasing laser pulse number, i.e. increasing Ag coverage. We found that changing only this experimental parameter the peak surface plasmon resonance absorption of the nanoparticles can be correlated with their geometrical characteristics and tuned through the UV-visible spectrum. As a second step the established experimental conditions were used to obtain nanostructures consisting of anatase phase $\mathrm{TiO}_{2}$ thin films covered by $\mathrm{Ag}$ nanoparticles. $\mathrm{TiO}_{2}$ photacatalysis has been extensively studied for environmental protection applications, decomposition of organic pollutants in water and air. ${ }^{30-32}$ However, the large band gap or $\mathrm{TiO}_{2}$ and quick recombination of the photogenerated change carriers severely limits practical applications. Addition of metal nanoparticles acting as electron traps could represent a solution to overcome this inconvenience. About the presence of metal particles on the surface of oxide catalysts many controversial results are reported and photocatalytic activity has been found to depend on both the preparation method and the nature of the degraded pollutant. ${ }^{33-35}$ Development of synthesis methods ensuring the precise control on nanoparticles size and uniform distribution on the catalyst surface, as well as the understanding of the implied photoinduced mechanisms are essential for practical applications. In this study the optimal conditions were determined for the creation of $\mathrm{Ag}-\mathrm{TiO}_{2}$ nanocomposites with improved photocatalitic activity as compared to anatase phase $\mathrm{TiO}_{2}$ through the established relationship between the Ag nanoparticles morphological characteristics and deposition parameters.

\section{Experimental}

$\mathrm{Ag}$ nanoparticles and nanostructures consisting of $\mathrm{TiO}_{2}$ thin films covered by $\mathrm{Ag}$ nanoparticles have been grown inside a stainless steel reaction chamber. $\mathrm{A} \mathrm{KrF}^{*}$ excimer laser $\left(\lambda=248 \mathrm{~nm}, \tau_{\mathrm{FWHM}} \cong 25 \mathrm{~ns}, v=10 \mathrm{~Hz}\right)$ was used for the irradiation of $\mathrm{Ag}$ (99.9 \% purity) and $\mathrm{TiO}_{2}$ (99.8\% purity) targets (Aldrich). The oxide targets were prepared from $\mathrm{TiO}_{2}$ powders by pressing at $3 \mathrm{MPa}$. The obtained pellets were sintered at $1100{ }^{\circ} \mathrm{C}$ for 4 hours. The laser beam incidence angle onto the target was chosen of about $45^{\circ}$. The incident laser fluence on the target surface was set at about $2 \mathrm{~J} / \mathrm{cm}^{2}$. The 
complete PLD workstation was purchased from SURFACE \& SURFACE systems \& technology GmbH \& Co KG, Hückelhoven, DE.

For the growth of the Ag nanoparticles the number of the subsequent laser pulses was varied in the range (20-1000) for each deposition experiment. To avoid piercing, the targets were both rotated with a frequency of $3 \mathrm{~Hz}$ and translated in the X-Y surface plane during the multipulse laser irradiation. Before each experiment the reaction chamber was evacuated with a high vacuum installation down to a residual pressure of $10^{-6} \mathrm{~Pa}$. The dynamic ambient gas pressure during the irradiations was kept at $10 \mathrm{~Pa}$ by feeding high-purity oxygen (99.999\%) into the chamber with the aid of a calibrated gas inlet. An MKS 100 controller was used for the measurement of the gas pressures.

The (001) $\mathrm{SiO}_{2}$ quartz substrates were placed parallel to the target at a separation distance of $5 \mathrm{~cm}$, and heated during the nanoparticles synthesis process at a temperature value of $500{ }^{\circ} \mathrm{C}$. A ramp of $20^{\circ} \mathrm{C} / \mathrm{min}$ was chosen for heating the substrates to reach the deposition temperature. Once the nanostructures growth was completed, the cooling down to room temperature was performed in the same reactive ambient gas atmosphere as used for the nanostructures growth, with a ramp of $20^{\circ} \mathrm{C} / \mathrm{min}$.

A number of 4500 subsequent laser pulses were used for the growth of the oxide thin film. All experimental parameters, target rotation and translation, $\mathrm{SiO}_{2}$ substrates temperature and heating regime, target to substrate separation distance, as well as ambient gas nature and pressure were identical to those used for the synthesis of the $\mathrm{Ag}$ nanoparticles.

Prior to introduction inside the reaction chamber, the targets and substrates were carefully cleaned in ultrasonic bath with ethanol. Additional target cleaning was achieved with the application of preliminary laser pulses for the elimination of the last contaminants and impurities present on the target surface. During the laser cleaning procedure a shutter was interposed at the mid-distance between the target and the substrate, parallel to them. 
The surface morphology and growth mode of the deposited nanostructures were investigated by atomic force microscopy (AFM) in acoustic (dynamic) configuration with an Agilent 5100 apparatus and field emission scanning electron microscopy (FESEM) with a Fei Quanta 650 FEG apparatus. The crystalline structure of the deposited thin films was investigated by grazing incidence angle X-ray diffraction (GIXRD). The measurements were performed with a Bruker Advance D-8 system coupled with a Gadds detector $(\mathrm{Cu} \mathrm{K} \alpha, \lambda=1.5418 \AA$ ). X-ray photoelectron spectroscopy (XPS) studies were performed with a SPECS EA10P hemispherical analyzer using a nonmonochromatized X-ray source ( $\mathrm{Al} \mathrm{K \alpha}$ line of $1486.6 \mathrm{eV}$ and $300 \mathrm{~W}$ ), placed perpendicular to the analyzer axis and calibrated using the $1 \mathrm{~s}$ line of $\mathrm{C}$. The measurements were made in ultra high vacuum (UHV) at residual pressure around $10^{-8}$ $\mathrm{Pa}$. The optical absorbance measurements were performed with a ThermoSpectronic, Helios Gamma spectrophotometer in the wavelength range of 300-800 $\mathrm{nm}$. The photocatalytic activity of the deposited $\mathrm{Ag}-\mathrm{TiO}_{2}$ nanostructures with $1 \mathrm{~cm}^{2}$ surface area was studied by measuring the concentration changes in time of organic methylene blue dye in aqueous solution. A monochromatic $365 \mathrm{~nm}$ wavelength, $125 \mathrm{~W} \mathrm{Hg}$ lamp was used for irradiations. During the photodegradation experiments the absorbance of the solution was measured at $665 \mathrm{~nm}$ wavelength which corresponds to the peak absorbance of methylene blue in the UV-visible spectral range, with a ThermoSpectronic, Helios Gamma apparatus. The samples were immersed in $30 \mathrm{ml}$ solutions with $10^{-5} \mathrm{M}$ initial dye concentration. The intensity of the light emitted by the lamp on the sample surface was $0.2 \mathrm{~mW} / \mathrm{cm}^{2}$. Fig. 1 shows a schematic diagram of the photoreactor consisting of the $\mathrm{Hg}$ lamp, water refrigerated vessel, and magnetic stirrer placed in an aluminium housing to prevent interfering of external light in the photodegradation experiments.

3. Results and discussions

Fig. 2a-d contains the AFM images of the Ag nanoparticles obtained with different number of laser pulses applied for the irradiation of the Ag targets. The formation of spherical shape nanoparticles can be clearly distinguished. Both nanoparticles diameter as well as height increase with the increase of the number of laser pulses, i.e. with the 
amount of the deposited material. The several bigger, few tens of $\mathrm{nm}$ sized particles visible on the surface of the samples are possibly due to the coalescence processes. In order to have a reference we provided the AFM image of an uncovered $\mathrm{SiO}_{2}$ quartz substrate (Fig. 2e). As can be observed, the surface is smooth, with a local height not exceeding $1.5 \mathrm{~nm}$. The calculated minimum root-mean square (RMS) surface roughness of the $\mathrm{SiO}_{2}$ substrate is around $0.13 \mathrm{~nm}$.

Fig. 3 shows the profiles corresponding to the uncovered $\mathrm{SiO}_{2}$ quartz substrate as well as the $\mathrm{Ag}$ nanoparticles deposited on the substrate surface with increasing number of laser pulses. As can be observed the height of the nanoparticles increases with the increase of the number of laser pulses. An average height of about $2.4 \mathrm{~nm}$ corresponding to the sample obtained with 20 laser pulses and around $10 \mathrm{~nm}$ at 200 pulses applied for the ablation of the Ag target were calculated on 500x500 $\mathrm{nm}^{2}$ AFM scan areas. The histograms of nanoparticles diameters evaluated on the same AFM scan areas are presented in Fig. 4. The average diameter of the grains increases gradually from about $5 \mathrm{~nm}$ corresponding to the samples obtained with 20 pulses (Fig. 4a), to about $23 \mathrm{~nm}$ for the sample obtained with 200 pulses (Fig. 4d). Table I summarizes the estimated values concerning particles mean diameter, mean height, density as well as RMS surface roughness as a function of number of incident laser pulses. The density of the particles decreases while the RMS surface roughness value increases gradually due to the formation of larger size particles with the increase the number of laser pulses. At low Ag content the nanoparticles are well separated and have a spherical in-plane shape with a narrow size distribution following a Volmer-Weber growth regime on the substrate surface. However, as reported, their size distribution becomes broader as the Ag content increases due to coarsening and coalescence of the NCs. ${ }^{27,36}$

The nanoparticles obtained with the low, up to 200 subsequent laser pulses applied for the ablation of the Ag targets are well separated and the average diameter and height could correctly be estimated. However, for higher number of pulses the gaps between the particles are on the order of the AFM tip diameter $(\sim 6 \mathrm{~nm})$. Consequently, the AFM tip can not reach the substrate surface between the particles, and therefore the maximum height determined from the images could be underestimated. The real shape and inplane dimensions of the particles can be observed on the FE-SEM micrographs as a 
function of number of laser pulses (Fig. 5). The micrographs confirm a formation of well separated nanoparticles on the substrate surface, with a quite homogeneous size distribution, controlled by the number of subsequent laser pulses. We note that the very small particles obtained with 20 subsequent laser pulses could not be visualised by SEM. Conversely, at higher number of pulses, until 200 the values estimated for the mean diameters of the nanoparticles by AFM corroborates well with those obtained by FE-SEM if the influence of the AFM tip on the measurements is taken into account. In order to reduce the widening effect, much more pronounced in the measurements of the particles in-plane dimensions as compared to their height, ${ }^{37}$ we used a geometric deconvolution ${ }^{38}$ considering a spherical tip with a radius of $6 \mathrm{~nm}$. The mean particle diameters after deconvolution are in agreement with the TEM results (Table I). With the further increase of the number of pulses the increase of the coupling between the nanoparticles leads to a gradual change of the reflected color from blue to metallic, ${ }^{39}$ as occurred in our experiments at number of pulses exceeding 400.

We present in Fig. 6 the GIXRD diffractograms of the Ag nanoparticles obtained with increasing number of laser pulses. The diffractograms reproduce the patterns of polycrystalline cubic Ag phase, with lines at $38.1^{\circ}$ corresponding to the most intense (111) and at $44.3^{\circ}$ to the (200) lattice plane reflections, as referred to in the JCPDS Card No. 00-004-0783. ${ }^{40}$ As can be seen, the lines intensity increases with the increase of the number of laser pulses due to increase of the Ag coverage.

The XPS spectrum of silver nanoparticles obtained with 100 subsequent laser pulses is presented in Fig. 7. The $\mathrm{Ag} 3 \mathrm{~d}$ region consists of two main peaks belonging to the $\mathrm{Ag}$ $3 \mathrm{~d} 5 / 2$ and $\mathrm{Ag} 3 \mathrm{~d} 3 / 2$ doublet. The $\mathrm{Ag} 3 \mathrm{~d} 5 / 2$ peak position at $368.57 \mathrm{eV}$ is in good agreement with the values of $368.00,368.26$, or $368.40 \mathrm{eV}$ reported in Refs. [41], [42], and [43], respectively for metallic silver. The low intensity satellite at $364.67 \mathrm{eV}$ is typical for non-monochromatized $\mathrm{Al} \mathrm{K \alpha}$ excitation source. ${ }^{44}$ We note that the shape of the XPS spectrum and peak positions is similar for all samples, independently on the number of subsequent laser pulses applied for the ablation of the Ag targets.

Under visual inspection the aspect of the samples indicated the formation of Ag clusters instead of a continuous metallic Ag layer. Their color was changing from nearly transparent at 20, to light yellow at 60 , orange at 100 , and finally dark blue at 200 laser 
pulses, as a function of their morphology and density on the substrate surface. As known, color variation arises from changes in the size, shape and proximity to each other of nanoparticles. ${ }^{45}$ The UV-visible optical absorption spectra of the Ag nanoparticles obtained with increasing number of laser pulses are presented in Fig. 8. The absorption maximum around $440 \mathrm{~nm}$ appearing in the spectrum of the nanoparticles obtained with 60 subsequent laser pulses (Fig. 8b) is associated with the surface plasmon resonance (SPR) absorption band, characteristic to metallic nanoparticles. The absoption is attributed to the conversion of incident photons of this wavelength to surface plasmons as a result of interaction with the free electron distribution on the silver nanoparticles surface. ${ }^{1}$ Approximately at the same wavelength an absorption band appears also in the case of the nanoparticles obtained with 20 laser pulses (Fig. 8a). Its very low intensity could be attributed to the reduced number of Ag particulates on the substrate surface. The absorption maximum shifts towards higher wavelengths with the increase of the number of laser pulses. The shift can be associated with the increase of the nanoparticles size, i. e. in-plane diameter, with the increase of the Ag coverage. ${ }^{46,47}$ Moreover, an additional absorption shoulder appears at around $550 \mathrm{~nm}$ in the case of the sample obtained with 100 laser pulses (Fig. 8c). The absorption spectrum of the structure deposited at 200 pulses (Fig. 8d) is formed by a broad band in the visible spectral region. The additional absorption shoulder at higher wavelengths and the broadening of the main absorption band could be due to the coalescence of the nanoparticles with the increase of the Ag coverage. ${ }^{39,48}$

As a second step in our investigations we used the established experimental conditions for the synthesis of $\mathrm{Ag}$ nanoparticles to obtain nanostructures consisting of $\mathrm{TiO}_{2}$ thin films covered by Ag nanoparticles, for photocatalytic applications. The diffractogram of a reference titanium oxide thin film in a $2 \theta$ range which contains also the main reflections of the metallic $\mathrm{Ag}$ is composed by lines at $37.8^{\circ}$ and $38.6^{\circ}$ corresponding to the (004) and (112) lattice plane reflections of the tetragonal anatase $\mathrm{TiO}_{2}$ phase, as referred to in the JC-PDS Card No. $21-1272^{40}$ (Fig. 9a). The insert shows a scan on a wider $2 \theta$ range, composed by lines at $25.4^{\circ}, 37.8^{\circ}, 38.6^{\circ}$, and $55.1^{\circ}$ attributed to the most intense (101), (004), (112), as well as (211) lattice plane reflections of the polycrystalline tetragonal anatase $\mathrm{TiO}_{2}$ phase, with [112] preferred orientation. Stabilization of the metastable anatase crystal structure is essential as it is known that possesses higher photoactivity as compared to the thermodynamically stable rutile 
phase $\mathrm{TiO}_{2}{ }^{49}$ The line at $50.8^{\circ}$ corresponds to the (001) $\mathrm{SiO}_{2}$ substrate. An average size of $\mathrm{TiO}_{2}$ nanocrystallites of about $25 \mathrm{~nm}$ was determined by the Scherrer equation: ${ }^{50} \mathrm{D}_{\mathrm{hkl}}$ $=0.9 \lambda / \beta_{\mathrm{hkl}} \cos \theta_{\mathrm{hkl}}$, where $\lambda$ is the X-ray wavelength, $\theta_{\mathrm{hkl}}$ is the Bragg diffraction angle, and $\beta_{\mathrm{hkl}}$ is the full width at half-maximum (FWHM) in radian of the diffraction line corresponding to the most intense (112) lattice plane reflection.

In the diffractograms of the oxide thin films covered by Ag nanoparticles the shoulder appearing at $38.1^{\circ}$ assigned to the most intense (111) lattice plane reflection was the first indication of the presence of the metallic Ag, for the sample obtained with 100 laser pulses incident on the Ag target (Fig. 9c). With the increase of the number of pulses the lines at $38.1^{\circ}$ and $44.3^{\circ}$ corresponding to the (111) and (200) lattice plane reflections, as referred to in the JC-PDS Card No. 00-021-1272, ${ }^{40}$ become clearly visible (Fig. 9d-f).

The photocatalytic activity of the $\mathrm{TiO}_{2}-\mathrm{Ag}$ nanostructures was examined through the decrease of methylene blue dye concentration, $\mathrm{C}$, of the solution in time. The obtained results are presented in Fig. 10. The reference shown in Fig. 10 represents the methylene blue dye self-degradation process in time. $\mathrm{C}_{\mathrm{o}}$ stands for the initial dye concentration. No significant difference was observed in the photocatalytic activity of the samples containing low Ag loading, obtained with 60 (Fig. 10b) and 100 (Fig. 10c) subsequent laser pulses incident on the $\mathrm{Ag}$ targets, as compared to the bare $\mathrm{TiO}_{2}$ thin film (Fig. 10a). On the contrary, at number of pulses exceeding 200 (Fig. 10d,e) faster photodegradation rate of the organic dye was achieved than in the case of the pure $\mathrm{TiO}_{2}$ catalyst (Fig. 10a). We would like to note that the bare $\mathrm{TiO}_{2}$ thin film had a thickness of about $400 \mathrm{~nm}$, as determined by cross-sectional scanning electron microscopy investigations. The corresponding catalytic amount is around $5 \mathrm{mg} / \mathrm{l}$. Moreover, corroborating the AFM and FE-SEM data the Ag amount was calculated and found to be between 0.6 and $1.8 \mu \mathrm{g} / \mathrm{cm}^{2}$ for increasing number of pulses from 60 to 200 . These values corroborates well with those estimated using the growth rates measured by X-ray reflectometry 0.09 as well as $0.008 \mathrm{~nm} /$ pulse for $\mathrm{TiO}_{2}$ and $\mathrm{Ag}$, respectively.

As known, the photocatalytic reaction takes place on the surface of the catalysts and recombination of photogenerated electrons and holes is very fast. The enhanced 
photoreactivity can be explained by the transfer of photogenerated electrons from $\mathrm{TiO}_{2}$ to the metal particles, inhibiting electron-hole recombination and thus raising the oxidation efficiency of the positive holes generated in the valence band of $\mathrm{TiO}_{2}{ }^{51,52}$ Metal nanoparticles can act as electron traps due to the formation of a Schottky barrier at the metal-semiconductor contact. ${ }^{52}$ As a consequence the holes, having strong oxidative power, can decompose organic substances more efficiently. Moreover, the tail of the localized surface plasmon resonance in the near-UV region can have an effect on the photocatalytic activity. ${ }^{53-55}$ Surface plasmon resonance absorption is associated with a significant enhancement of the electric near-field in the vicinity of the Ag NPs. It was reported that the enhanced near-field could boost the excitation of electron-hole pairs in $\mathrm{TiO}_{2}$ and therefore increase the efficiency of the photocatalysis. ${ }^{54,55}$

Our results demonstrate that there is an optimal loading amount of Ag nanoparticles on $\mathrm{TiO}_{2}$. Indeed, at number of pulses higher than 400 the photocatalytic activity of the samples decreased (Fig. 10f). This feature could be attributed to the gradual reduction of the active $\mathrm{TiO}_{2}$ surface area directly irradiated by the UV light with the increase of the Ag coverage, causing lower photocatalytic efficiency.

\section{Conclusions}

$\mathrm{Ag}$ nanoparticles and $\mathrm{Ag}-\mathrm{TiO}_{2}$ nanostructures were synthesized by pulsed laser deposition technique. The established dependence between the Ag nanoparticles morphology and SPR absorption permits the continuous tuning of their optical properties in the UV-visible spectral range. The obtained results demonstrate that laser techniques allows for the creation of $\mathrm{Ag}-\mathrm{TiO}_{2}$ nanocomposites with improved photocatalytic activity as compared to bare $\mathrm{TiO}_{2}$. The optimum loading amount of $\mathrm{Ag}$ nanoparticles was determined. It was found that there exists a maximum threshold for the $\mathrm{Ag}$ loading value after which the photocatalytic activity of the $\mathrm{Ag}-\mathrm{TiO}_{2}$ nanocomposites decreases. The sequential nature of the technique including pulsed laser radiation permits the precise control of the size, distribution and amount of noble metal nanoparticles deposited on the oxide photocatalysts surface. It was proposed that two distinct mechanisms can contribute to the enhanced photoreactivity under near-UV irradiation. Ag NPs retard electron-hole recombination by photogenerated electron 
transfer from $\mathrm{TiO}_{2}$. Moreover, localised surface plasmon resonance absorption of $\mathrm{Ag}$ NPs can have positive effect on the photocatalytic activity.

\section{Acknowledgement}

Financial support from the Ministry of Science and Innovation of the Spanish Government MAT2008-04931, CSD2008-00023, the Spanish National Research Council 200860I211, and the Catalan Government (Departament de Medi Ambient i Habitatge), SGR-0333 is acknowledged with thanks. 
References

1. U. Kreibig and M. Vollmer, "Optical Propeties of Metal Clusters", Springer, New York, 1995.

2. "Optical Propeties of Nanostructured Random Media", edited by V. M. Shalaev, Springer, New York, 2002.

3. D. Bedeaux and J. Vlieger, "Optical Properties of Surfaces", Imperial College Press, London, 2002.

4. S. Shrivastava, T. Bera, S. K. Singh, G. Singh, P. Ramachandrarao, and D. Dash, "Characterization of Antiplatelet Properties of Silver Nanoparticles", ACS Nano 3 [6] 1357-1364 (2009).

5. I. Sur, D. Cam, M. Kahraman, A. Baysal, and M. Culha, "Interaction of multifunctional silver nanoparticles with living cells", Nanotechnology 21 [17] 175104 (2010).

6. M. M. Kemp, A. Kumar, S. Mousa, E. Dyskin, M. Yalcin, P. Ajayan, R. J. Linhardt, and S. A. Mousa, "Gold and silver nanoparticles conjugated with heparin derivative possess anti-angiogenesis properties", Nanotechnology 20 [45] 455104 (2009).

7. J. N. Anker, W. P. Hall, O. Lyandres, N. C. Shah, J. Zhao, and R. P. Van Duyne, "Biosensing with plasmonic nanosensors", Nature Materials 7 [6] 442-453 (2008).

8. S. S. Kim, S. I. Na, J. Jo, D. Y. Kim, and Y. C. Nah, "Plasmon enhanced performance of organic solar cells using electrodeposited Ag nanoparticles”, Appl. Phys. Lett. 93 [7] 073307 (2008).

9. I. Ahmed, C. E. Png, E. P. Li, and R. Vahldieck, "Electromagnetic wave propagation in a Ag nanoparticle-based plasmonic power divider", Opt. Express 17 [1] 337-345 (2009).

10. B. Mukherjee and M. Mukherjee, "Nonvolatile memory device based on Ag nanoparticle: Characteristics improvement”, Appl. Phys. Lett. 94 [17] 173510 (2009).

11. H. Wang and L. Qi, "Controlled Synthesis of $\mathrm{Ag}_{2} \mathrm{~S}, \mathrm{Ag}_{2} \mathrm{Se}$, and $\mathrm{Ag}$ Nanofibers by Using a General Sacrificial Template and Their Application in Electronic Device Fabrication”, Adv. Funct. Mater. 18 [8] 1249-1256 (2008).

12. R. Sato-Berru, R. Redon, A. Vazquez-Olmos, and J. M. Saniger, "Silver nanoparticles synthesized by direct photoreduction of metal salts. Application in 
surface-enhanced Raman spectroscopy”, J. Raman Spectrosc. 40 [4] 376-380 (2009).

13. A. Mayer and M. Antonietti, "Investigation of polymer-protected noble metal nanoparticles by transmission electron microscopy: control of particle morphology and shape”, Colloid Polym. Sci. 276 [9] 769-779 (1998).

14. Y. Deng, Y. Sun, P. Wang, D. Zhang, X. Jiao, H. Ming, Q. Zhang, Y. Jiao, X. Sun, "Nonlinear optical properties of silver colloidal solution by in situ synthesis technique", Current Appl. Phys. 8 [1] 13-17 (2008).

15. P. V. Kazakevich, A. V. Simakin, V. V. Voronov, and G. A. Shafeev, "Laser induced synthesis of nanoparticles in liquids”, Appl. Surf. Sci. 252 [13] 4373-4380 (2006).

16. H. Han, Y. Fang, and Z. Li, H. Xu, “Tunable surface plasma resonance frequency in Ag core/Au shell nanoparticles system prepared by laser ablation”, Appl. Phys. Lett. 92 [2] 023116 (2008).

17. G. Compagnini, E. Messina, R.S. Cataliotti, A. Grillo, and G. Giaquinta, "Diffusion dynamics of laser-ablated noble-metal nanoparticles in liquids", Phil. Mag. Lett. 89 [4] 250-256 (2009).

18. V. Amendola, S. Polizzi, and M. Meneghetti, "Laser Ablation Synthesis of Gold Nanoparticles in Organic Solvents", J. Phys. Chem. B 110 [14] $7232-7237$ (2006).

19. T. R. Jensen, M. D. Malinsky, C. L. Haynes, and R. P. Van Duyne, "Nanosphere Lithography: Tunable Localized Surface Plasmon Resonance Spectra of Silver Nanoparticles”, J. Phys. Chem. B 104 [45] 10549-10556 (2000).

20. H. Pan, S. H. Ko, and C. P. Grigoropoulos, "The coalescence of supported gold nanoparticles induced by nanosecond laser irradiation", Appl. Phys. A: Mater. Sci. \& Process. 90 [2] 247-253 (2008).

21. R. Dolbec, E. Irissou, M. Chaker, D. Guay, F. Rosei, and M. A. El Khakani, "Growth dynamics of pulsed laser deposited Pt nanoparticles on highly oriented pyrolitic graphite substrates”, Phys. Rev. B 70 [20] 201406(R) (2004).

22. M. Hirai and A. Kumar, "Wavelength tuning of surface plasmon resonance by annealing silver-copper nanoparticles”, J. Appl. Phys. 100 [1] 014309 (2006).

23. C. Agrafiotis, A. Tsetsekou, C. J. Stournaras, A. Julbe, L. Dalmazio, C. Guizard, G. Boretto, M. Debenedetti, and F. Parussa, "Evaluation of sol-gel methods for the synthesis of doped-ceria environmental catalysis systems: Part II. Catalytic activity and resistance to thermal aging”, Appl. Catal. B: Environmental, 34 [2] 149-159 (2001). 
24. K. Zakrzewska, M. Radecka, A. Kruk, and W. Osuch, "Noble metal/titanium dioxide nanocermets for photoelectrochemical applications", Solid State Ionics 157 [14] 349-356 (2003).

25. C. W. Lai, J. An, and H. C. Ong, "urface-plasmon-mediated emission from metalcapped ZnO thin films” Appl. Phys. Lett. 86 [25] 251105 (2005).

26. Y. K. Mishra, S. Mohapatra, D. Kabiraj, B. Mohanta, N. P. Lalla, J. C. Pivin, and D. K. Avasthi, "Synthesis and characterization of Ag nanoparticles in silica matrix by atom beam sputtering”, Scripta Materialia 56 [7] 629-632 (2007).

27. J. Gonzalo, R. Serna, J. Solis, D. Babonneau, and C. N. Afonso, "Morphological and interaction effects on the surface plasmon resonance of metal nanoparticles", J. Phys: Condens. Matter 15 [42] S3001-S3010 (2003).

28. E. György, J. Santiso, A. Figueras, A. Giannoudakos, M. Kompitsas, and I. N. Mihailescu, "Morphology evolution and local electric properties of Au nanoparticles on ZnO thin films", J. Appl. Phys. 98 [8] 084302 (2005).

29. E. György, G. Sauthier, A. Figueras, A. Giannoudakos, M. Kompitsas, and I. N. Mihailescu, "Growth of $\mathrm{Au}-\mathrm{TiO}_{2}$ nanocomposite thin films by a dual-laser, dual-target system”, J. Appl. Phys. 100 [11] 114302 (2006).

30. H. M. Martin, "Photodegradation of Water Pollutants", CRC Press: Boca Raton, FL, 1996.

31. Y. Shiraishi, N. Saito, and T. Hirai, "Adsorption-Driven Photocatalytic Activity of Mesoporous Titanium Dioxide”, J. Am. Chem. Soc. 127 [37], 12820-12822 (2005).

32. X. X. Yang, C. D. Cao, L. Erickson, K. Hohn, R. Maghirang, and K. Klabunde, "Synthesis of visible-light-active $\mathrm{TiO}_{2}$-based photocatalysts by carbon and nitrogen doping", J. Catal. 260 [1], 128-133 (2008).

33. C. He, Y. $\mathrm{Yu}, \mathrm{X} . \mathrm{Hu}$, and A. Larbot, "Influence of silver doping on the photocatalytic activity of titania films”, Appl. Surf. Sci. 200 [1-4], 239-247 (2002).

34. H. Keskinen, J. M. Makela, M. Aromaa, J. Keskinen, S. Areva, C. V. Teixeira,J. B. Rosenholm, V. Pore, M. Ritala, M. Leskela, M. Raulio, M. S. Salkinoja-Salonen, E. Levanen, and T. Mantyla, "Titania and titania-silver nanoparticle deposits made by Liquid Flame Spray and their functionality as photocatalyst for organic- and biofilm Renoval”, Catal. Lett. Vol. 111 [ 3-4], 127-132 (2006).

35. W. Y. Teoha, L. Mädler, and R. Amal, "Inter-relationship between Pt oxidation states on $\mathrm{TiO}_{2}$ and the photocatalytic mineralisation of organic matters", J. Catal. 251 
[2], 271-280 (2007).

36. J. P. Barnes, A. K. Petford-Long, R. C. Doole, R. Serna, J. Gonzalo, A. SuarezGarcia, C. N. Afonso, and D. Hole, "Structural studies of Ag nanocrystals embedded in amorphous $\mathrm{Al}_{2} \mathrm{O}_{3}$ grown by pulsed laser deposition", Nanotechnology 13 [4], 465-470 (2002).

37. C. Hulteen, D. A. Treichel, M. T. Smith, M. L. Duval, T. R. Jensen, and R. P. Van Duyne, Nanosphere Lithography: Size-Tunable Silver Nanoparticle and Surface Cluster Arrays, J. Phys. Chem. B 103 [19], 3854-3863 (1999).

38. D. Keller, Reconstruction of STM and AFM images distorted by finite-size tips, Surf. Sci. 253 [1-3], 353-364 (1991).

39. P. Mulvaney, L. M. Liz-Marzan, M. Giersig, and T. Ung, "Silica encapsulation of quantum dots and metal clusters", J. Mater. Chem. 10 [6], 1259-1270 (2000).

40. International Centre for Diffraction Data: Newton Square, PA, 2010.

41. M. Mathew, R. Jayakrishnan, P. M. Ratheesh Kumar, C. Sudha Kartha, and K. P. Vijayakumar, "Anomalous behavior of silver doped indium sulfide thin films", J. Appl. Phys. 100 [3] 033504 (2006).

42. R. Romand, M. Roubin, and J. P. Deloume, "X-ray photoelectron emission studies of mixed selenides AgGaSe 2 and $\mathrm{Ag}_{9} \mathrm{GaSe}_{6}$ ”, J. Solid State Chem. 25 [1] 59-64 (1978).

43. M. P. Seah, G. C. Smith, and M. T. Anthony, "AES: Energy calibration of electron spectrometers. I-an absolute, traceable energy calibration and the provision of atomic reference line energies", Surf. Interface Anal. 15 [5] 293-308 (1990).

44. C. D. Wagner, W. M. Riggs, L. E. Davis, J. F. Moulder, and G. E. Muilenberg, "Handbook of X-ray Photoelectron Spectroscopy", Perkin-Elmer Corporation, Physical Electronics Division, Eden Prairie, Minn. 1979.

45. L. M. Liz-Marzán, "Nanometals: formation and color”, Mater. Today, 7 [2], 26-31 (2004).

46. U. Pal, J. Garcia-Serrano, G. Casarrubias-Segura, N. Koshizaki, T. Sasaki, and S. Terahuchi, "Structure and optical properties of $\mathrm{M} / \mathrm{ZnO}(\mathrm{M}=\mathrm{Au}, \mathrm{Cu}, \mathrm{Pt})$ nanocomposites”, Solar En. Mater. \& Solar Cells 81 [3] 339-348 (2004).

47. D. M. Schaadt, B. Feng, and E. T. Yu, "Enhanced semiconductor optical absorption via surface plasmon excitation in metal nanoparticles”, Appl. Phys. Lett. 86 [6] 063106 (2005).

48. S. V. Roth, H. Walter, M. Burghammer, C. Riekel, B. Lengeler, C. Schroeder, M. Kuhlmann, T. Walther, A. Sehrbrock, R. Domnick, and P. Muller-Buschbaum, 
"Combinatorial investigation of the isolated nanoparticle to coalescent layer transition in a gradient sputtered gold nanoparticle layer on top of polystyrene", Appl. Phys. Lett. 88 [2] 021910 (2006).

49. N. Ruzycki, G.S. Herman, L.A. Boatner, and U. Diebold, "Scanning tunneling microscopy study of the anatase (1 00 ) surface”, Surf. Sci. Lett. 529 [1-2] L239-L244 (2003).

\section{D. B. Cullity (1978) "Elements of X-ray diffraction”, Addison Wesley, Reading.}

51. J. Zhang, L. Xiao, Y. Cong, and M. Anpo, "Preparation and Characterization of Multi-functional Titanium Dioxide Photocatalysts", Top Catal. 47 [3-4] 122-130 (2008).

52. Z. Liu, B. Guo, L. Hong, and H. Jiang, "Physicochemical and photocatalytic characterizations of $\mathrm{TiO}_{2} / \mathrm{Pt}$ nanocomposites", J. Photochem. Photobiol. A Chem. 172 [1] 81-88 (2005).

53. C. Hu, T. Peng, S. Hu, Y. Nie, X. Zhou, J. Qu, and H. He, "Plasmon-Induced Photodegradation of Toxic Pollutants with $\mathrm{Ag}-\mathrm{AgI} / \mathrm{Al}_{2} \mathrm{O}_{3}$ under Visible-Light Irradiation", J. Am. Chem. Soc. 132 [2] 857-862 (2010).

54. T. Hirakawa and P. V. Kamat, "Charge Separation and Catalytic Activity of Ag$\mathrm{TiO}_{2}$ Core-Shell Composite Clusters under UV-Irradiation”, J. Am. Chem. Soc. 127 [11] 3928-3934 (2005).

55. K. Awazu, M. Fujimaki, C. Rockstuhl, J. Tominaga, H. Murakami, Y. Ohki, N. Yoshida, and T. Watanabe, "A Plasmonic Photocatalyst Consisting of Silver Nanoparticles Embedded in Titanium Dioxide”, J. Am. Chem. Soc. 130 [5] 1676-1680 (2008). 
Table I. Particles mean diameter, mean height, density, and surface RMS roughness calculated from 500x500 $\mathrm{nm}^{2}$ AFM scan areas as well as particles mean diameter calculated from FE-SEM micrographs as a function of number of incident laser pulses.

\begin{tabular}{|c|c|c|c|c|c|}
\hline $\begin{array}{c}\text { Number of } \\
\text { pulses }\end{array}$ & $\begin{array}{c}\text { Mean diameter } \\
\text { AFM [nm] }\end{array}$ & $\begin{array}{c}\text { Mean } \\
\text { height [nm] }\end{array}$ & $\begin{array}{c}\text { Density } \\
{\left[\mu \mathrm{m}^{-2}\right]}\end{array}$ & $\begin{array}{c}\text { RMS } \\
\text { roughness } \\
{[\mathrm{nm}]}\end{array}$ & $\begin{array}{c}\text { Mean diameter } \\
\text { FE-SEM [nm] }\end{array}$ \\
\hline 20 & 5 & 2.4 & 5700 & 0.6 & - \\
\hline 60 & 16 & 5.0 & 3600 & 1.2 & 10 \\
\hline 100 & 19 & 6.0 & 2900 & 1.3 & 14 \\
\hline 200 & 23 & 10.0 & 1900 & 2.2 & 18 \\
\hline
\end{tabular}


Figure captions

Fig. 1. Experimental set-up used for the photodegradation of organic methylene blue dye.

Fig. 2. AFM images of Ag nanoparticles obtained with (a) 20, (b) 60, (c) 100, and (d) 200 subsequent laser pulses as well as (e) (001) $\mathrm{SiO}_{2}$ quartz plate used as substrate for the growth of the nanoparticles.

Fig. 3. Surface profiles of (a) (001) $\mathrm{SiO}_{2}$ quartz plate and $\mathrm{Ag}$ nanoparticles obtained with (a) 20, (b) 60, (c) 100, as well as (d) 200 subsequent laser pulses

Fig. 4. Histograms of Ag nanoparticles diameters counted on $25 \mu \mathrm{m}^{2}$ surface areas of samples obtained with (a) 20, (b) 60, (c) 100, and (d) 200 subsequent laser pulses.

Fig. 5. FE-SEM images of Ag nanoparticles obtained with (a) 60, (b) 100, and (c) 200 subsequent laser pulses.

Fig. 6. GIXRD of Ag nanoparticles obtained with (a) 20, (b) 60, (c) 100, and (d) 200 subsequent laser pulses.

Fig. 7. XPS spectrum of Ag nanoparticles obtained with 100 subsequent laser pulses.

Fig. 8. UV-visible optical absorbance spectra of Ag nanoparticles obtained with (a) 20, (b) 60, (c) 100, and (d) 200 subsequent laser pulses.

Fig. 9. XRD of (a) $\mathrm{TiO}_{2}$ thin film as well as nanostructures consisting of $\mathrm{TiO}_{2}$ thin films and Ag nanoparticles obtained with (b) 60, (c) 100, (d) 200, (e) 400, and (f) 1000 subsequent laser pulses. The insert shows wider $\theta-2 \theta$ scan of the $\mathrm{TiO}_{2}$ thin film. 
Fig. 10. Comparison of methylene blue dye degradation process on (a) $\mathrm{TiO}_{2}$ thin film as well as nanostructures consisting of $\mathrm{TiO}_{2}$ thin films and $\mathrm{Ag}$ nanoparticles obtained with (b) 60, (c) 100, (d) 200, (e) 400, and (f) 1000 subsequent laser pulses, as a function of exposure time. The reference corresponds to the methylene blue dye self-degradation process in time. 


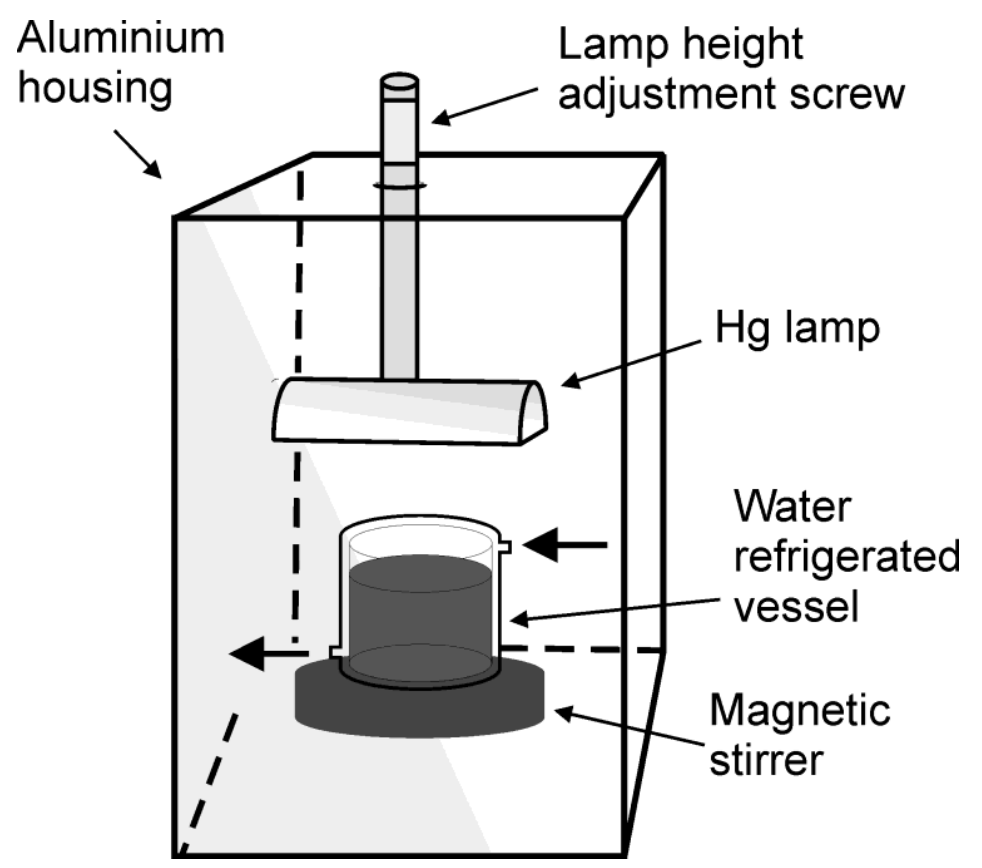

Fig. 1. 


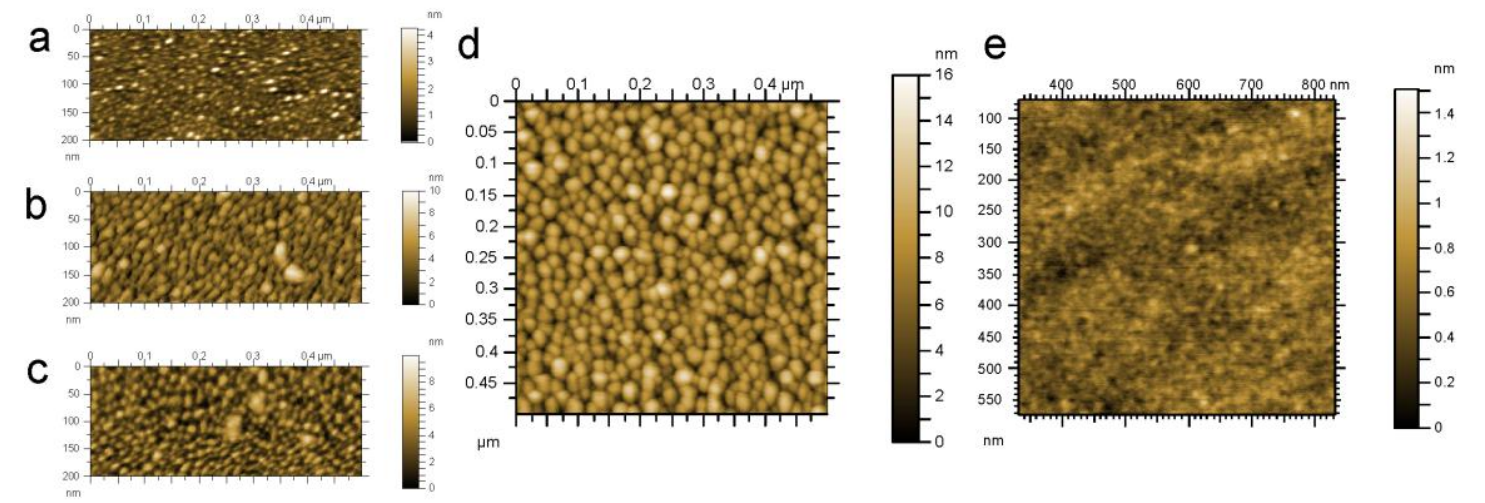

Fig. 2. 


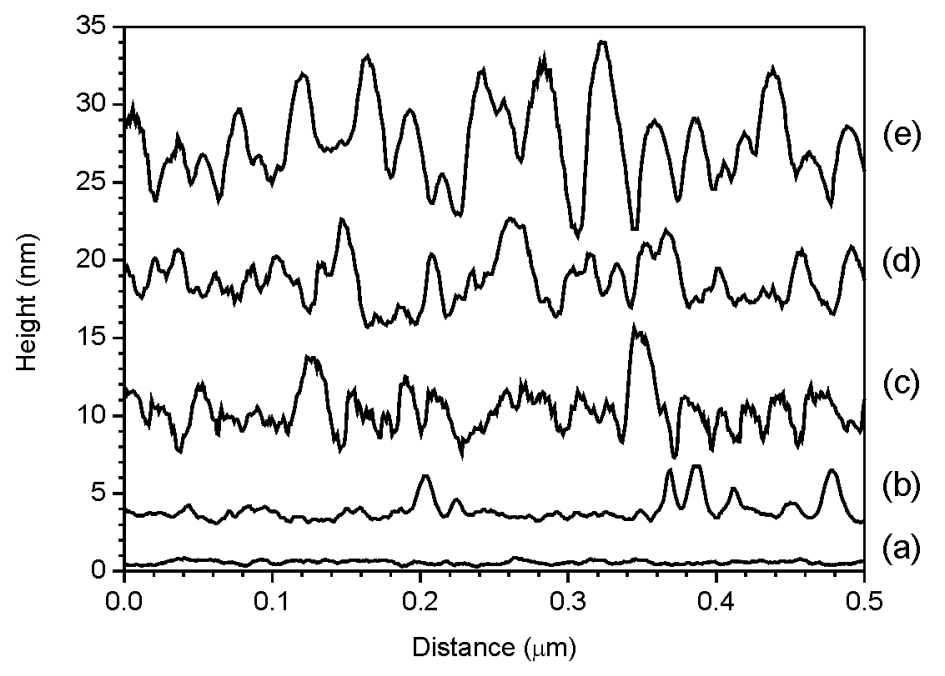

Fig. 3. 

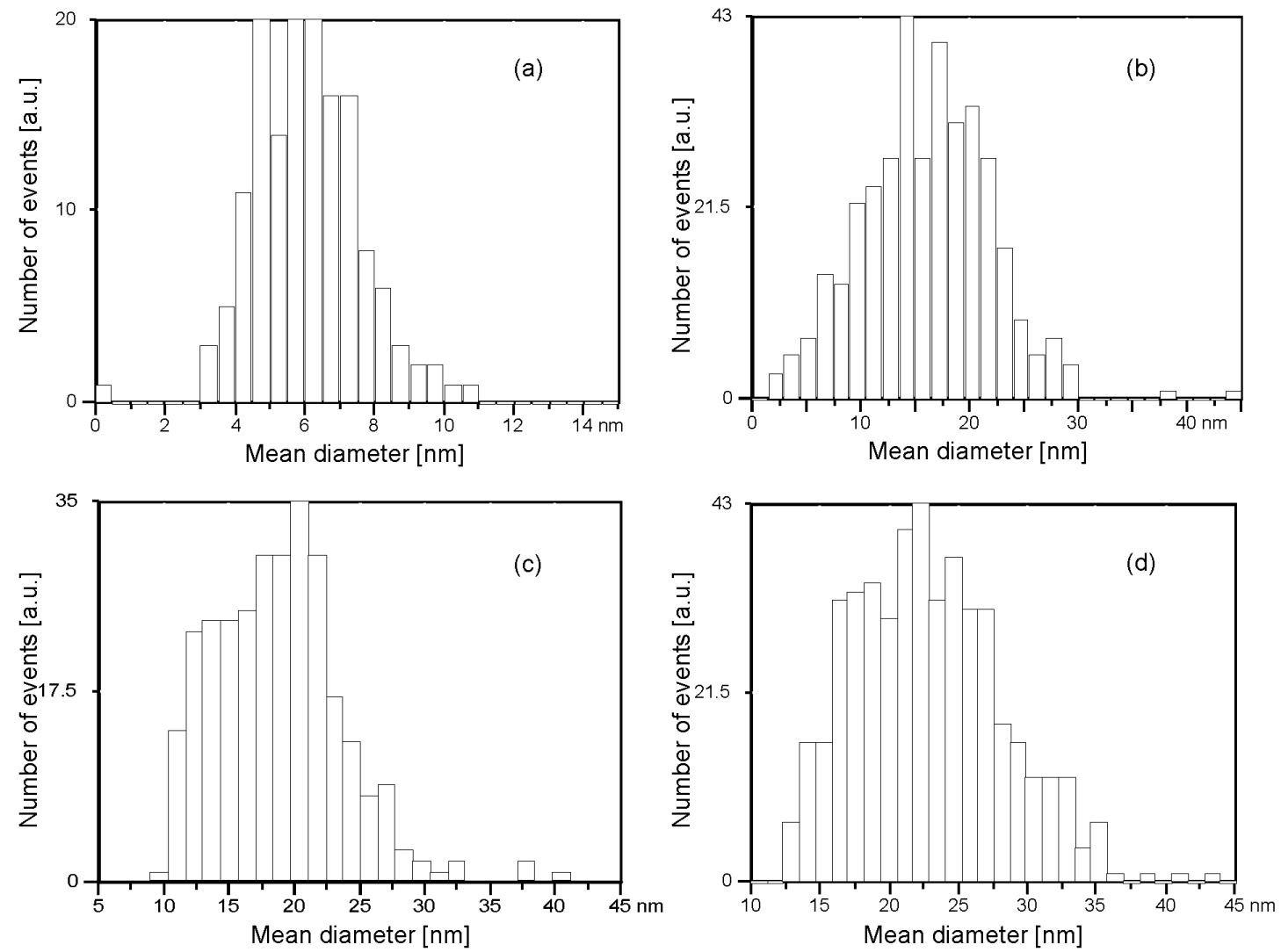

Fig. 4. 

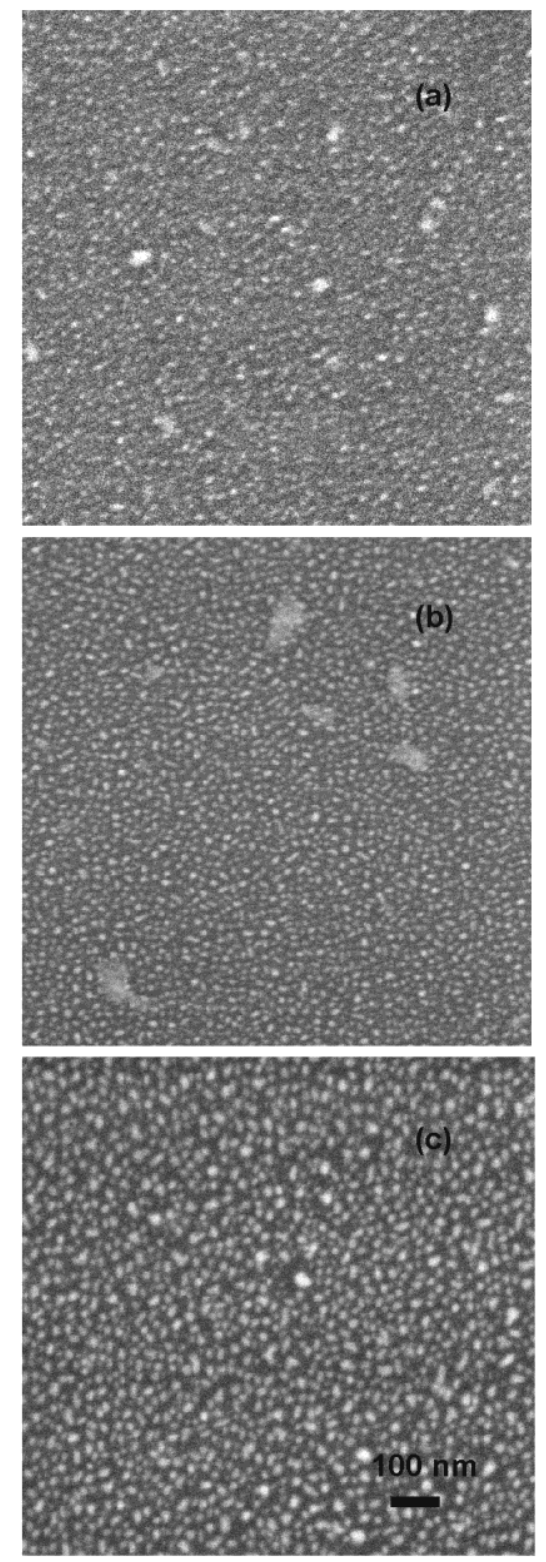

Fig. 5. 


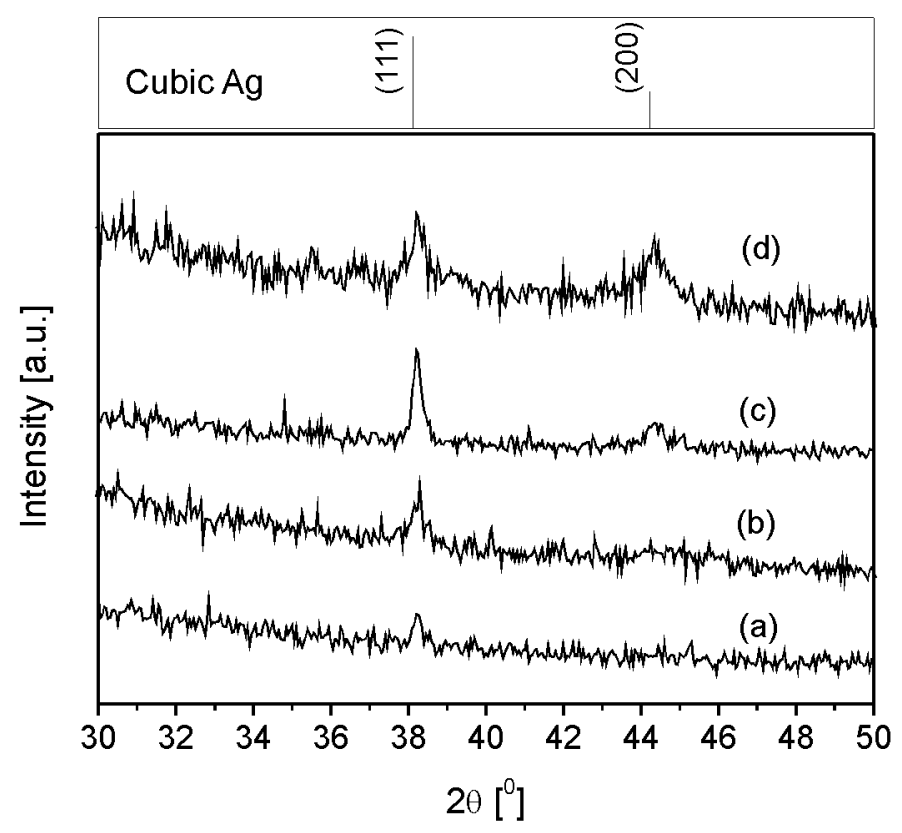

Fig. 6. 


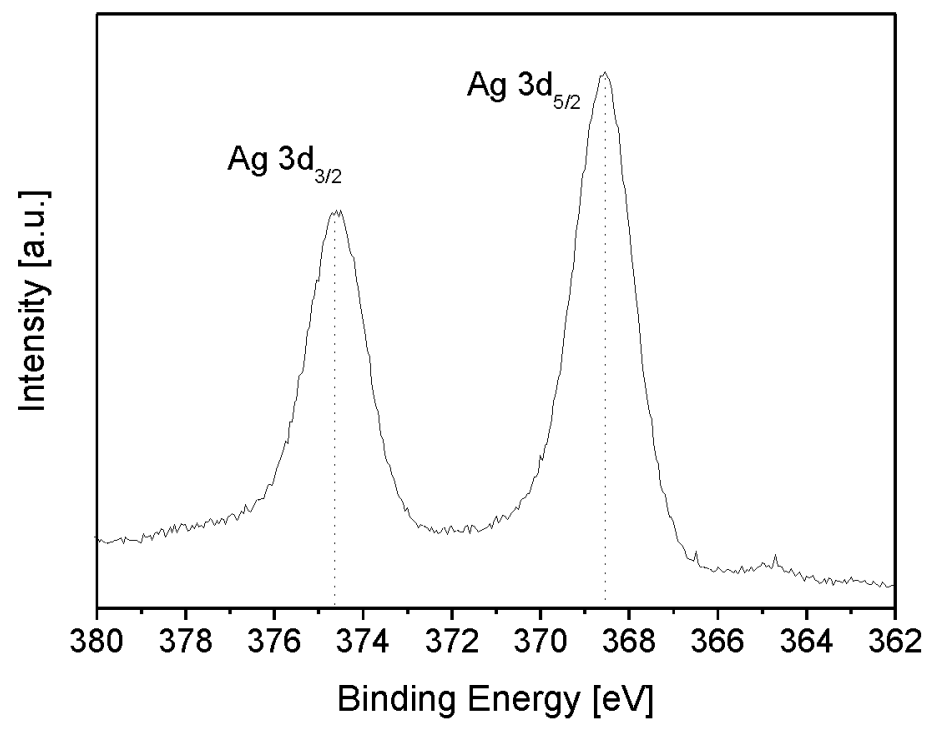

Fig. 7. 


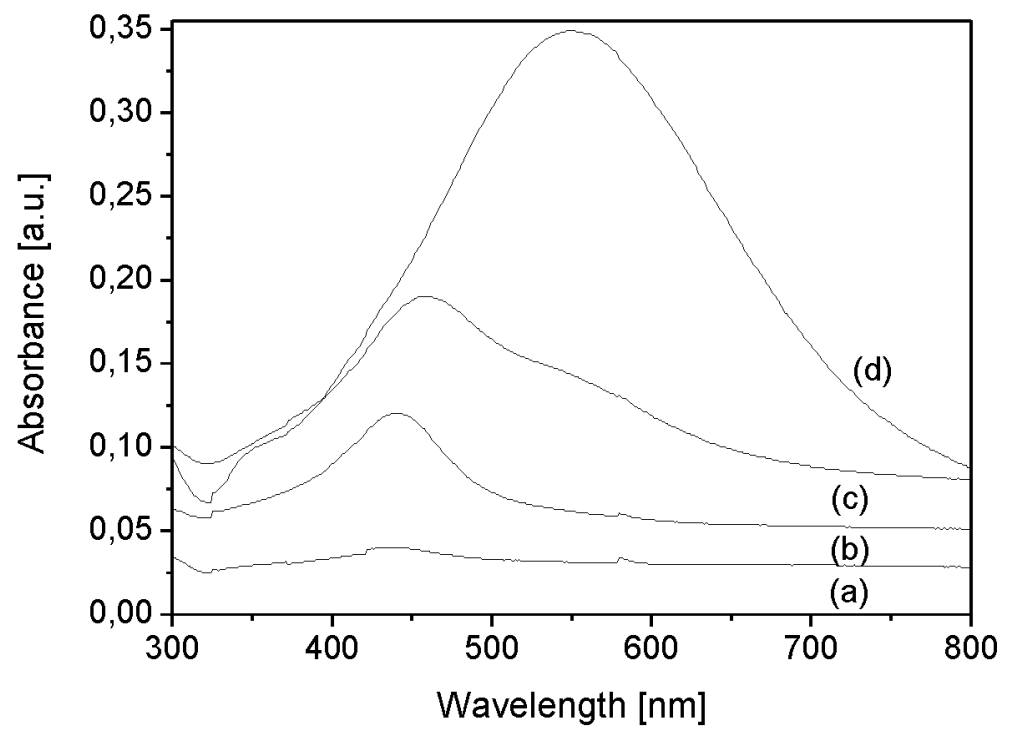

Fig. 8. 


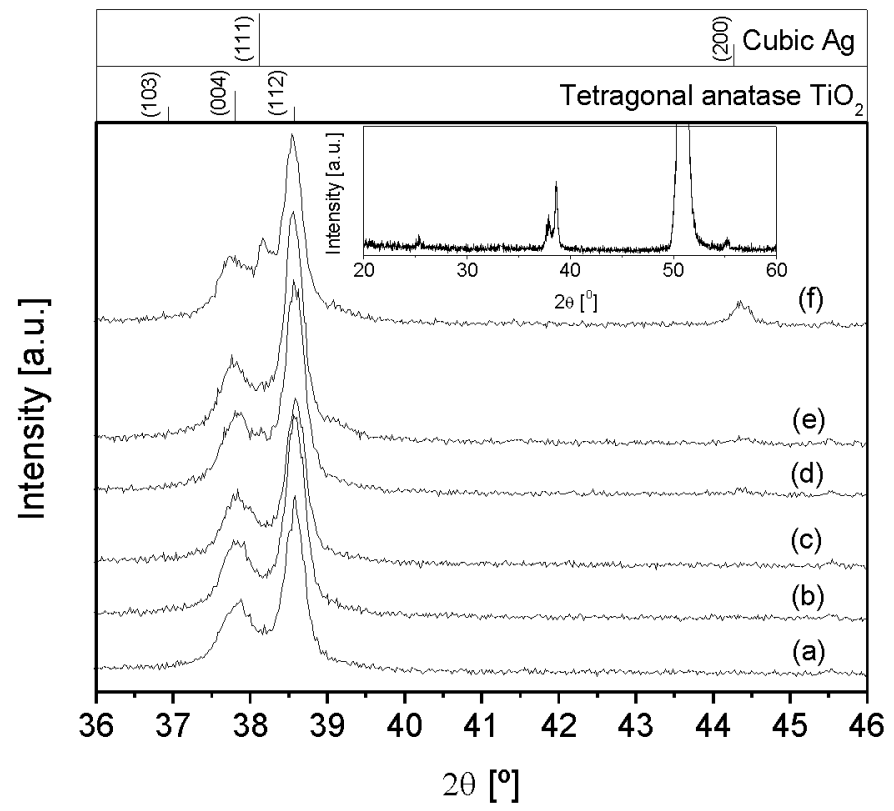

Fig. 9. 


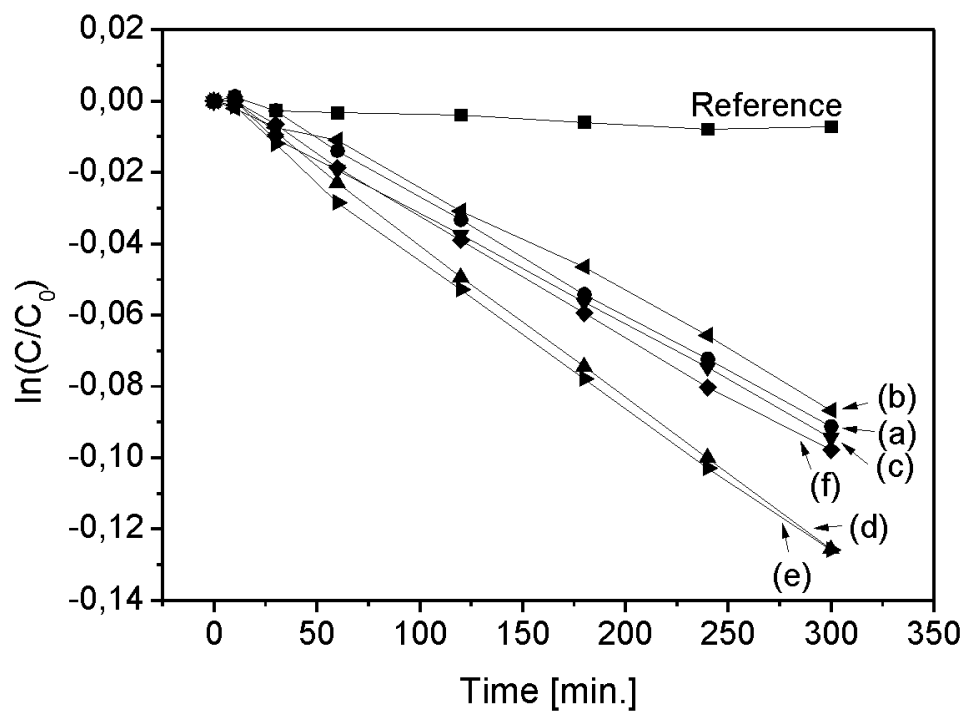

Fig. 10. 